\title{
Sub-Riemannian geodesics on the three-dimensional solvable non-nilpotent Lie group $\mathrm{SOLV}^{-}$
}

\author{
Mazhitova A.D. *
}

\section{Introduction}

In this paper we describe the geodesics of a left-invariant sub-Riemannian metric on the three-dimensional solvable Lie group Sol.

This group is widely known in geometry, because it allows compact quotient-spaces and it gives one of the Thurston three-dimensional geometries [1]. By the classification theorem of Agrachev-Barilari [2] there are invariant sub-Riemannian geometries realized on four solvable non-nilpotent Lie groups: $\mathrm{SE}(2), \mathrm{SH}(2), \mathrm{SOLV}^{-}$, and $\mathrm{SOLV}^{+}$.

In this classification, our geometry corresponds to the case $\mathrm{SOLV}^{-}$:

$$
\mathrm{Sol}=\mathrm{SOLV}^{-} \text {. }
$$

The case of $\mathrm{SOLV}^{+}$we shall consider separately.

Various aspects of the integration of geodesic flows on sub-Riemannian manifolds has been widely studied (see, for example, [3, 4, 5, 6, 12]). Note, that the geodesics of other three-dimensional nonsolvable or nilpotent subRiemannian geometries have been described recently in terms of elementary functions [7, 8]. In our situation it is necessary to use elliptic functions.

We thank I.A. Taimanov for posing the problem and Ya.V. Bazaikin for helpful discussions.

* Mechanical-Mathematical Department, Al-Farabi Kazakh National University, AlFarabi ave. 71, Almaty 050038, Kazakhstan; e-mail: Akmaral.Mazhitova@kaznu.kz 


\section{Basic definitions}

\subsection{Geodesics of sub-Riemannian manifolds}

Let $M^{n}$ be a smooth $n$-dimensional manifold. A smooth family of $k$-dimensional subspaces in the tangent spaces to points of $M^{n}$

$$
\Delta=\left\{\Delta(q): \quad \Delta(q) \in T_{q} M^{n} \quad \forall q \in M^{n}, \quad \operatorname{dim} \Delta(q)=k\right\}
$$

is called completely nonintegrable, if the vector fields tangent to $\Delta$, and all their iterated commutators generate the tangent bundle $T M^{n}$ :

$\operatorname{span}\left\{\left[f_{1},\left[\ldots\left[f_{m-1}, f_{m}\right] \ldots\right]\right](q): f_{i}(p) \in \Delta(p) \quad \forall p \in M^{n}, m=1, \ldots\right\}=T_{q} M^{n}$.

Sometimes this distribution is called completely nonholonomic.

A two-dimensional distributions in a three-dimensional manifold is completely nonholonomic if and only if

$$
\operatorname{span}\left\{f_{1}(q), f_{2}(q),\left[f_{1}(q), f_{2}(q)\right]\right\}=T_{q} M^{3},
$$

where at every point $q$ the vectors $f_{1}(q)$ and $f_{2}(q)$ form a basis in $\Delta(q)$.

Let $g_{i j}$ be a complete Riemannian metric on $M^{n}$. A triple $\left(M^{n}, \Delta, g_{i j}\right)$ is called a sub-Riemannian manifold. A Lipschitz continuous curve $\gamma$ : $[0, T] \rightarrow M^{n}$ is called admissible if $\dot{\gamma}(t) \in \Delta(\gamma(t))$ for almost all $t \in[0, T]$. The length of this curve is equal to

$$
l(\gamma)=\int_{0}^{T} \sqrt{g_{\gamma(t)}(\dot{\gamma}(t), \dot{\gamma}(t))} d t
$$

The distance between two points on the manifold is defined by formula

$$
d\left(q_{0}, q_{1}\right)=\inf _{\gamma \in \Omega_{q_{0}, q_{1}}} l(\gamma)
$$

where $\Omega_{q_{0}, q_{1}}$ is the set of all admissible curves connecting points $q_{0}$ and $q_{1}$. This function $d(\cdot, \cdot)$ is called the sub-Riemannian metric on $M^{n}$. A geodesic of this metric is an admissible curve $\gamma:[0, T] \rightarrow M^{n}$, which locally minimizes the length functional $l(\gamma)$.

Geodesics of sub-Riemannian metrics satisfy the Pontryagin maximum principle (see, for instance, [5]), which we formulate below. Let $f_{1}, \ldots, f_{k}$ be vector fields which are tangent to $\Delta$ and span $\Delta$ at every point of $M^{n}$ (or of a domain of $M^{n}$ ).

The Pontryagin maximum PRInciple is as follows: 
- Let $M^{n}$ be a smooth $n$-dimensional manifold. Let us consider for Lipschitz continuous curves the following minimum problem

$\dot{q}=\sum_{i=1}^{k} u_{i} f_{i}(q), \quad u_{i} \in \mathbb{R}, \int_{0}^{T} \sum_{i=1}^{k} u_{i}^{2}(t) d t \longrightarrow \min , q(0)=q_{0}, q(T)=q_{1}$

with a fixed $T$. Let us consider the mapping $\mathcal{H}: T^{*} M^{n} \times \mathbb{R} \times \mathbb{R}^{k} \rightarrow \mathbb{R}$, given by the function

$$
\mathcal{H}\left(q, \lambda, p_{0}, u\right):=\left\langle\lambda, \sum_{i=1}^{k} u_{i} f_{i}(q)\right\rangle+p_{0} \sum_{i=1}^{k} u_{i}^{2}
$$

If a curve $q(\cdot):[0, T] \rightarrow M^{n}$ with a control $u(\cdot):[0, T] \rightarrow \mathbb{R}^{k}$ is optimal, then there exists Lipshitzian covector function $\lambda(\cdot): t \in$ $[0, T] \mapsto \lambda(t) \in T_{q(t)}^{*} M^{n},\left(\lambda(t), p_{0}\right) \neq 0$ and a constant $p_{0} \leq 0$ such that

i) $\dot{q}(t)=\frac{\partial \mathcal{H}}{\partial \lambda}\left(q(t), \lambda(t), p_{0}, u(t)\right)$,

ii) $\dot{\lambda}(t)=-\frac{\partial \mathcal{H}}{\partial q}\left(q(t), \lambda(t), p_{0}, u(t)\right)$,

iii) $\frac{\partial \mathcal{H}}{\partial u}\left(q(t), \lambda(t), p_{0}, u(t)\right)=0$.

A curve $q(\cdot):[0, T] \rightarrow M^{n}$, satisfying the Pontryagin maximum principle is called an extremal (curve). To such a curve there corresponds a set of pairs $\left(\lambda(\cdot), p_{0}\right)$. The type (normal or abnormal) of an extremal depends on the value of $p_{0}$ :

- if $p_{0} \neq 0$, then the extremal is called normal;

- if $p_{0}=0$, then the extremal is called abnormal;

- extremal is called strictly abnormal if it is not projected (on $M^{n}$ ) onto a normal extremal.

For a normal extremal we may put $p_{0}=-\frac{1}{2}$.

Normal extremals are geodesics [5]. In the contact case, when at every point the distribution $\Delta$ coincides with the annihilator of the contact form on $M^{n}$, there are no nontrivial abnormal extremals (this fact is indicated in [7]). In the case, when the space of vector fields on a manifold is generated by vector fields tangent to the nonholonomic distribution and their 
commutators, there are no strictly abnormal extremals [5]. We recall, that an extremal is called strictly abnormal, if its projection on $M^{n}$ does not coincide with the projection of any normal extremal. Both of the above statements apply to three-dimensional sub-Riemannian manifold $M^{3}$.

By iii), $u_{i}=\left\langle\lambda(t), f_{i}(t)\right\rangle$ and a curve $q(\cdot):[0, T] \rightarrow M^{n}$ is geodesic if and only if it is the projection onto $M^{n}$ of a solution $(\lambda(t), q(t))$ of a Hamiltonian system on $T^{*} M^{n}$ with the following Hamiltonian function:

$$
H(\lambda, q)=\frac{1}{2}\left(\sum_{i=1}^{k}\left\langle\lambda, F_{i}\right\rangle^{2}\right), \quad q \in M^{n}, \quad \lambda \in T_{q}^{*} M^{n} .
$$

The Hamiltonian $H$ is constant along any solution of the Hamiltonian system. Moreover, $H=\frac{1}{2}$ if and only if the geodesic is length parameterized.

\subsection{Elliptic functions. Jacobi functions}

Let us recall some necessary facts of Jacobi elliptic functions. The integrals

$$
\int_{0}^{x} \frac{d x}{\sqrt{\left(1-x^{2}\right)\left(1-k^{2} x^{2}\right)}}
$$

and

$$
\int_{0}^{x} \frac{\sqrt{1-k^{2} x^{2}}}{\sqrt{\left(1-x^{2}\right)}} d x
$$

are called elliptic integrals of first and second kind, respectively, in the normal Legendre form (see [10, 13]), where $k(0<k<1)$ is the modulus of these integrals, $k^{\prime}=\sqrt{1-k^{2}}$ is the additional modulus. By the substitution $x=\sin \varphi$ these integrals reduce to the normal trigonometric form

$$
\begin{gathered}
F(\varphi, k)=\int_{0}^{\varphi} \frac{d \alpha}{\sqrt{\left(1-k^{2} \sin ^{2} \alpha\right)}}=\int_{0}^{\sin \varphi} \frac{d x}{\sqrt{\left(1-x^{2}\right)\left(1-k^{2} x^{2}\right)}}, \\
\mathrm{E}(\varphi, k)=\int_{0}^{\varphi} \sqrt{1-k^{2} \sin ^{2} \alpha} d \alpha=\int_{0}^{\sin \varphi} \frac{\sqrt{1-k^{2} x^{2}}}{\sqrt{1-x^{2}}} d x .
\end{gathered}
$$

Consider an integral of first kind in the normal trigonometric form

$$
v=\int_{0}^{\varphi} \frac{d \varphi}{\sqrt{1-k^{2} \sin ^{2} \varphi}}
$$


Now consider the upper limit as a function of $v$. This function is denoted by

$$
\varphi=\operatorname{am}(v, k)=\operatorname{am} v
$$

and is called the amplitude, and this process is called an inversion of the integral. Thus, next functions:

$$
\begin{gathered}
\sin \varphi=\sin (\operatorname{am} v)=\operatorname{sn} v, \\
\cos \varphi=\cos (\operatorname{am} v)=\operatorname{cn} v, \\
\Delta \operatorname{am} v=\sqrt{1-k^{2} \sin ^{2} \varphi}=\sqrt{1-k^{2} \operatorname{sn}^{2} v}=\operatorname{dn} v
\end{gathered}
$$

are called the Jacobi functions and are related by

$$
\operatorname{sn}^{2} v+\mathrm{cn}^{2} v=1, \quad \mathrm{dn}^{2} v+k^{2} \operatorname{sn}^{2} v=1 .
$$

By derivation, we obtain

$$
\begin{gathered}
\frac{d \operatorname{sn} v}{d v}=\operatorname{cn} v \operatorname{dn} v \\
\frac{d \operatorname{cn} v}{d v}=-\operatorname{sn} v \operatorname{dn} v \\
\frac{d \operatorname{dn} v}{d v}=-k^{2} \operatorname{sn} v \operatorname{cn} v
\end{gathered}
$$

and conclude, that

$$
\begin{aligned}
& \left(\frac{d \operatorname{sn} v}{d v}\right)^{2}=\left(1-\operatorname{sn}^{2} v\right)\left(1-k^{2} \operatorname{sn}^{2} v\right) \\
& \left(\frac{d \operatorname{cn} v}{d v}\right)^{2}=\left(1-\operatorname{cn}^{2} v\right)\left(k^{2}+k^{2} \operatorname{cn}^{2} v\right) \\
& \left(\frac{d \operatorname{dn} v}{d v}\right)^{2}=\left(1-\operatorname{dn}^{2} v\right)\left(\operatorname{dn}^{2} v-k^{2}\right)
\end{aligned}
$$

The first equation of (3) implies that $\operatorname{sn} v$ is the inversion of the first kind elliptic integral in the normal Legendre form

$$
v=\int_{0}^{\operatorname{sn} v} \frac{d x}{\sqrt{\left(1-x^{2}\right)\left(1-k^{2} x^{2}\right)}} .
$$


From the second and third equations obtained that $\operatorname{cn} v$ and $\operatorname{dn} v$ are the result of conversion of next functions

$$
\begin{gathered}
v=\int_{1}^{\mathrm{cn} v} \frac{d x}{\sqrt{\left(1-x^{2}\right)\left(k^{\prime 2}+k^{2} x^{2}\right)}} \\
v=\int_{1}^{\operatorname{dn} v} \frac{d x}{\sqrt{\left(1-x^{2}\right)\left(x^{2}-k^{\prime 2}\right)}} .
\end{gathered}
$$

All Jacobi functions are periodic. Note that the function $\operatorname{sn} v$ is odd, but $\operatorname{cn} v$ and $\operatorname{dn} v$ are even, therefore we assume, what in the two last integral, when the functions $\mathrm{cn} v$ and $\operatorname{dn} v$ pass through the critical points, respectively changes a sign of radical.

\section{Sub-Riemannian problem on the group $\mathrm{SOLV}^{-}$}

Let us consider the three-dimensional Lie group $\mathrm{SOLV}^{-}$formed by all matrices of the form

$$
\left(\begin{array}{ccc}
e^{-z} & 0 & x \\
0 & e^{z} & y \\
0 & 0 & 1
\end{array}\right), x, y, z \in \mathbb{R}
$$

Its Lie algebra is spanned by the vectors

$$
e_{1}=\left(\begin{array}{lll}
0 & 0 & 1 \\
0 & 0 & 0 \\
0 & 0 & 0
\end{array}\right), \quad e_{2}=\left(\begin{array}{lll}
0 & 0 & 0 \\
0 & 0 & 1 \\
0 & 0 & 0
\end{array}\right), \quad e_{3}=\left(\begin{array}{ccc}
-1 & 0 & 0 \\
0 & 1 & 0 \\
0 & 0 & 0
\end{array}\right),
$$

meeting the following commutation relations:

$$
\left[e_{1}, e_{2}\right]=0 ; \quad\left[e_{1}, e_{3}\right]=e_{1} ; \quad\left[e_{2}, e_{3}\right]=-e_{2} .
$$

We take a new basis

$$
a_{1}=e_{1}+e_{2} ; \quad a_{2}=e_{1}-e_{2} ; a_{3}=e_{3},
$$

in which the commutation relations take the form

$$
\left[a_{1}, a_{2}\right]=0, \quad\left[a_{1}, a_{3}\right]=a_{2}, \quad\left[a_{2}, a_{3}\right]=a_{1} .
$$

Let us consider a left-invariant metric on $\mathrm{SOLV}^{-}$, which is defined by its values in the unit of the group:

$$
\left(e_{i}, e_{j}\right)=\delta_{i j}
$$


The Lie group $\mathrm{SOLV}^{-}$is diffeomorphic to the space $\mathbb{R}^{3}$. Indeed, $x, y, z$ are the global coordinates on $\mathrm{SOLV}^{-}$and they also may be considered as global coordinates on $\mathbb{R}^{3}$. The tangent space at each point of $\mathrm{SOLV}^{-}$is spanned by matrices of the form

$$
\partial_{x}=\left(\begin{array}{lll}
0 & 0 & 1 \\
0 & 0 & 0 \\
0 & 0 & 0
\end{array}\right), \quad \partial_{y}=\left(\begin{array}{lll}
0 & 0 & 0 \\
0 & 0 & 1 \\
0 & 0 & 0
\end{array}\right), \quad \partial_{z}=\left(\begin{array}{ccc}
-e^{-z} & 0 & 0 \\
0 & e^{z} & 0 \\
0 & 0 & 0
\end{array}\right),
$$

which are the left translations of the basic vectors:

$$
L_{q *}\left(e_{1}\right)=e^{-z} \partial_{x}, \quad L_{q *}\left(e_{2}\right)=e^{z} \partial_{y}, \quad L_{q *}\left(e_{3}\right)=\partial_{z} .
$$

Since the metric is left-invariant, we have

$$
g_{i j}(x, y, z)=\left(\begin{array}{ccc}
e^{2 z} & 0 & 0 \\
0 & e^{-2 z} & 0 \\
0 & 0 & 1
\end{array}\right)
$$

For the basis $a_{1}, a_{2}, a_{3}$ we have

$$
L_{q *}\left(a_{1}\right)=e^{-z} \partial_{x}+e^{z} \partial_{y}, \quad L_{q *}\left(a_{2}\right)=e^{-z} \partial_{x}-e^{z} \partial_{y}, \quad L_{q *}\left(a_{3}\right)=\partial_{z} .
$$

The inner product takes the form

$$
\left\langle L_{q *}\left(a_{i}\right), L_{q *}\left(a_{j}\right)\right\rangle=\left\langle a_{i}, a_{j}\right\rangle=\left(\begin{array}{ccc}
2 & 0 & 0 \\
0 & 2 & 0 \\
0 & 0 & 1
\end{array}\right)
$$

In this paper we study the sub-Riemannian problem on the three-dimensional Lie group $\mathrm{SOLV}^{-}$defined by the distribution $\Delta=\operatorname{span}\left\{a_{1}, a_{3}\right\}$ with metric (8).

Let $G=\mathrm{SOLV}^{-}, \mathcal{G}$ be its Lie algebra with the basic vectors $a_{1}, a_{2}, a_{3}$ (17). We split the Lie algebra $\mathcal{G}$ into the sum sum $p \bigoplus k$, where $p=$ $\operatorname{span}\left\{a_{1}, a_{3}\right\}, \quad k=\operatorname{span}\left\{a_{2}\right\}$.

Let us consider a two-dimensional left-invariant distribution $\Delta=\operatorname{span}\left\{a_{1}, a_{3}\right\}$ in $T G$, and a left-invariant Riemannian metric (8) for which the spaces $p$ and $k$ are orthogonal, i.e., the metric tensor splits as follows:

$$
g=\left(g_{i j}\right)=g_{p}+g_{k}
$$

Let us introduce a parameter $\tau$ and consider the metrics

$$
g_{\tau}=g_{p}+\tau g_{k}
$$


Every such a metric together with $\Delta$ defines the same sub-Riemannian manifold because only the restriction of the metric onto $\Delta$ is important.

However the Hamiltonian function for the geodesic flows of these metrics depends on $\tau$ :

$$
H(x, p, \tau)=\frac{1}{2} g_{\tau}^{i j}(x) p_{i} p_{j},
$$

where $g_{i j} g^{j k}=\delta_{i}^{k}$. We have

$$
g_{\tau, i j}=\left(\begin{array}{ccc}
\frac{1+\tau}{2} e^{2 z} & \frac{1-\tau}{2} & 0 \\
\frac{1-\tau}{2} & \frac{1+\tau}{2} e^{-2 z} & 0 \\
0 & 0 & 1
\end{array}\right), \quad g_{\tau}^{i j}=\left(\begin{array}{ccc}
\frac{1+\tau}{2 \tau} e^{-2 z} & -\frac{1-\tau}{2} & 0 \\
-\frac{1-\tau}{2} & \frac{1+\tau}{2 \tau} e^{2 z} & 0 \\
0 & 0 & 1
\end{array}\right) .
$$

The Hamiltonian function $H$ for the normal geodesic flow of the sub-Riemannian metric is obtained from $H(x, p, \tau)$ in the limit

$$
\tau \rightarrow \infty
$$

and we derive

$$
H\left(x, y, z, p_{x}, p_{y}, p_{z}\right)=\frac{1}{4} e^{-2 z} p_{x}^{2}+\frac{1}{2} p_{x} p_{y}+\frac{1}{4} e^{2 z} p_{y}^{2}+\frac{1}{2} p_{z}^{2} .
$$

The Hamiltonian equations $\dot{x}^{i}=\frac{\partial H}{\partial p_{i}}, \dot{p}_{i}=-\frac{\partial H}{\partial x^{i}}$ take the form

$$
\begin{array}{ll}
\dot{x}=\frac{1}{2} e^{-2 z} p_{x}+\frac{1}{2} p_{y}, & \dot{p_{x}}=0, \\
\dot{y}=\frac{1}{2} e^{2 z} p_{y}+\frac{1}{2} p_{x}, & \dot{p_{y}}=0, \\
\dot{z}=p_{z}, & \dot{p_{z}}=\frac{1}{2} e^{-2 z} p_{x}^{2}-\frac{1}{2} e^{2 z} p_{y}^{2} .
\end{array}
$$

These differential equations can be derived from the Pontryagin maximum principle. The corresponding Hamiltonian takes the form

$H\left(x, y, z, p_{x}, p_{y}, p_{z}, p_{0}, u_{1}, u_{3}\right)=\frac{1}{\sqrt{2}}\left(u_{1} p_{x} e^{-z}+u_{1} p_{y} e^{z}\right)+u_{3} p_{z}+p_{0}\left(u_{1}^{2}+u_{3}^{2}\right)$,

where $p_{0}=-\frac{1}{2}, u_{1}, u_{3}$ are control functions. 
The system (10) has three first integrals:

$$
I_{1}=H, \quad I_{2}=p_{x}, \quad I_{3}=p_{y},
$$

which are functionally independent almost everywhere, and therefore the system is completely integrable.

Since the flow is left-invariant as well as the distribution $\Delta$ and the metric, without loss of generality we assume, that all geodesics originate at the unit of group, that is, we have the following initial conditions for the system (10):

$$
x(0)=0, \quad y(0)=0, \quad z(0)=0 .
$$

In the sequel, we put

$$
H=\frac{1}{2}, \quad \frac{p_{x}}{\sqrt{2}}=a, \quad \frac{p_{y}}{\sqrt{2}}=b
$$

By substituting these expressions into (9), we obtain

$$
1=\left(e^{-z} a+e^{z} b\right)^{2}+p_{z}^{2},
$$

which implies

$$
p_{z}= \pm \sqrt{1-\left(e^{-z} a+e^{z} b\right)^{2}} .
$$

By substituting this expression to the third equation of (10) we obtain equation for the temporal variable $t$ for positive values of $p_{z}$

$$
t=\int \frac{d z}{\sqrt{1-\left(e^{-z} a+e^{z} b\right)^{2}}} .
$$

If $p_{z}<0$, then all calculations will be similar, but with contrary sign.

Let us make the change of variables

$$
u=e^{z}
$$

and rewrite (13) as

$$
t=\int \frac{d u}{\sqrt{u^{2}-\left(a+b u^{2}\right)^{2}}} .
$$

The last expression is not integrated in terms of elementary functions and defines an elliptic integral, except of special cases, when this elliptic integral degenerates. These cases will be discussed below.

Let us consider the generic case $a \neq 0$ and $b \neq 0$. 
The subradical expression in (14) haves discriminant $D=1-4 a b \geq 0$ accordingly (12).

$D=0$ if and only if $p_{z}=0$ accordingly system (10) and equation (12). That case is degenerative.

Thus, if $D>0\left(a b<\frac{1}{4}\right)$, then there exist $\sigma_{1}^{2}$ and $\sigma_{2}^{2}$, such that the following

$$
\begin{gathered}
u^{2}-\left(a+b u^{2}\right)^{2}=-b^{2} u^{4}+(1-2 a b) u^{2}-a^{2}= \\
=-b^{2}\left(u^{2}-\sigma_{1}^{2}\right)\left(u^{2}-\sigma_{2}^{2}\right)=\sigma_{1}^{4} b^{2}\left(1-\frac{u^{2}}{\sigma_{1}^{2}}\right)\left(\frac{u^{2}}{\sigma_{1}^{2}}-\frac{\sigma_{2}^{2}}{\sigma_{1}^{2}}\right),
\end{gathered}
$$

and

$$
\sigma_{1,2}^{2}=\frac{1-2 a b \pm \sqrt{1-4 a b}}{2 b^{2}}
$$

Put

$$
w=\frac{u}{\sigma_{1}}
$$

and rewrite (14) in the following form

$$
t=\frac{1}{\sigma_{1} b} \int \frac{d w}{\sqrt{\left(1-w^{2}\right)\left(w^{2}-\frac{\sigma_{2}^{2}}{\sigma_{1}^{2}}\right)}} .
$$

We apply the Jacobi elliptic function (6) in order to inverse this integral:

$$
\sigma_{1} b t=\int_{1}^{\operatorname{dn}\left(\sigma_{1} b t\right)} \frac{d w}{\sqrt{\left(1-w^{2}\right)\left(w^{2}-\frac{\sigma_{2}^{2}}{\sigma_{1}^{2}}\right)}}
$$

where $k^{\prime 2}=\frac{\sigma_{2}^{2}}{\sigma_{1}^{2}}$. Therefore

$$
w=\operatorname{dn}\left(\sigma_{1} b t, k\right)
$$

where

$$
k^{2}=1-\frac{\sigma_{2}^{2}}{\sigma_{1}^{2}} .
$$

By inverting (16), putting $u=e^{z}$, and keeping in mind the initial condition (11) and the equality $\operatorname{dn}(0, k)=1$, we obtain

$$
z(t)=\ln \operatorname{dn}\left(\sigma_{1} b t, k\right) .
$$


By substituting this expression into the first equation of (10) and integrating it in elliptic functions (see[10]), we derive:

$x(t)=\frac{1}{\sqrt{2}}\left[\frac{a}{\sigma_{1} b}\left(-\frac{k^{2} \operatorname{sn}\left(\sigma_{1} b t\right) \mathrm{cn}\left(\sigma_{1} b t\right)}{k^{\prime 2} \sqrt{1-k^{2} \mathrm{sn}^{2}\left(\sigma_{1} b t\right)}}+\frac{1}{k^{\prime 2}} \mathrm{E}\left(\operatorname{am}\left(\sigma_{1} b t\right), k\right)\right)+b t\right]+C$,

where $\mathrm{E}(x, k)$ is elliptic integral of second kind (2) .

Since $\operatorname{sn}(0, k)=0, \operatorname{cn}(0, k)=1, \operatorname{am}(0, k)=0$ and $\mathrm{E}(0, k)=0$, we have $C=0$. From the second equation of this system we conclude that

$$
y(t)=\frac{1}{\sqrt{2}}\left(\frac{1}{\sigma_{1}} \mathrm{E}\left(\operatorname{am}\left(\sigma_{1} b t\right), k\right)+a t\right)+Q,
$$

with $Q=$ const. By (11), we compute that $Q=0$.

Let us now consider the cases, when the elliptic integral (14) degenerates:

1. $a=0, \quad b=0$;

2. $a=0, \quad b \neq 0$;

3. $b=0, \quad a \neq 0$;

4. $D=0\left(a b=\frac{1}{4}\right)$.

We consider its successively:

1) $a=0, b=0$. From the equations (10), (111) and (13) is cleaner, that

$$
x(t)=0, \quad y(t)=0, \quad z(t)=t .
$$

2) $a=0, \quad b \neq 0$. We have $p_{x}=0, \quad p_{y}=\sqrt{2} b$.

The equation (14) is rewritten as

$$
t=\int \frac{d u}{\sqrt{u^{2}-b^{2} u^{4}}} .
$$

By integration and transformation the resulting expression by the inverse change of variable, we obtain $u=e^{z}$ :

$$
e^{t}=\frac{C b e^{z}}{1+\sqrt{1-b^{2} e^{2 z}}},
$$

where $C=$ const and $C>0$. The last expression together with the initial condition (11) implies

$$
C=\frac{1+\sqrt{1-b^{2}}}{b},
$$


and we derive that

$$
e^{z}=\frac{2 C e^{t}}{b\left(C^{2}+e^{2 t}\right)}
$$

i.e.,

$$
z(t)=\ln \frac{2 C e^{t}}{b\left(C^{2}+e^{2 t}\right)},
$$

which after substituting the formula for $C$ takes the form

$$
z(t)=\ln \frac{2\left(1+\sqrt{1-b^{2}}\right) e^{t}}{2\left(1+\sqrt{1-b^{2}}\right)-b^{2}+b^{2} e^{2 t}} .
$$

By the first equation of (10), we have

$$
x(t)=\frac{b}{\sqrt{2}} t,
$$

and the second equation of (10) together with (20) and (11) implies

$$
y(t)=-\frac{\sqrt{2} C^{2}}{b\left(C^{2}+e^{2 t}\right)}+\frac{\sqrt{2} C^{2}}{b\left(C^{2}+1\right)} .
$$

Finally in the case $\mathbf{2}$ ) we have the explicit formulas for solutions:

$$
\begin{aligned}
& x(t)=\frac{b}{\sqrt{2}} t \\
& y(t)=-\frac{\sqrt{2}\left(2\left(1+\sqrt{1-b^{2}}\right)-b^{2}\right)}{2 b\left(1+\sqrt{1-b^{2}}\right)-b^{3}+b^{3} e^{2 t}}+\frac{\sqrt{2}\left(2\left(1+\sqrt{1-b^{2}}\right)-b^{2}\right)}{2 b\left(1+\sqrt{1-b^{2}}\right)} \\
& z(t)=\ln \frac{2\left(1+\sqrt{1-b^{2}}\right) e^{t}}{2\left(1+\sqrt{1-b^{2}}\right)-b^{2}+b^{2} e^{2 t}} . \\
& \text { 3) } b=0, \quad a \neq 0 . \text { We have } p_{y}=0, \quad p_{x}=\sqrt{2} a .
\end{aligned}
$$

The equation (14) takes the form

$$
t=\int \frac{d u}{\sqrt{u^{2}-a^{2}}}
$$

Let us put $u=e^{z}$ and derive

$$
e^{t}=\left(e^{z}+\sqrt{e^{2 z}-a^{2}}\right) C,
$$


where $C=$ const and $C>0$. The last expression together with (11) implies

$$
C=\frac{1}{1+\sqrt{1-a^{2}}}
$$

from which we obtain

$$
z(t)=\ln \frac{C^{2} a^{2}+e^{2 t}}{2 C e^{t}},
$$

where $C$ is given by (22). As in the case 1) we derive from the first two equations of (10) that

$$
x(t)=-\frac{\sqrt{2} a C^{2}}{e^{2 t}+C^{2} a^{2}}+\frac{\sqrt{2} a C^{2}}{1+C^{2} a^{2}}, \quad y(t)=\frac{a}{\sqrt{2}} t .
$$

Finally we obtain

$$
\begin{aligned}
& x(t)=-\frac{\sqrt{2} a}{e^{2 t}\left[2\left(1+\sqrt{1-a^{2}}\right)-a^{2}\right]+a^{2}}+\frac{\sqrt{2} a}{2\left(1+\sqrt{1-a^{2}}\right)}, \\
& y(t)=\frac{a}{\sqrt{2}} t \\
& z(t)=\ln \left(\frac{a^{2}}{2\left(1+\sqrt{1-a^{2}}\right) e^{t}}+\frac{\left(1+\sqrt{1-a^{2}}\right) e^{t}}{2}\right) .
\end{aligned}
$$

4) $D=0\left(a b=\frac{1}{4}\right)$.

Note that, the formula (12) by (11) is rewritten as

$$
(a+b)^{2}+p_{z}^{2}=1
$$

which means that

$$
|a+b| \leq 1
$$

Then it is clear that $a=b=\frac{1}{2}$ or $a=b=-\frac{1}{2}$, and for these values the equation (25) implies that $p_{z}=0$. Therefore solutions to (10) in the case 4) are linear:

$$
\begin{array}{r}
x(t)=\frac{t}{\sqrt{2}}, \quad y(t)=\frac{t}{\sqrt{2}}, \quad z(t)=0, \quad a=b=\frac{1}{2} ; \\
x(t)=-\frac{t}{\sqrt{2}}, \quad y(t)=-\frac{t}{\sqrt{2}}, \quad z(t)=0, \quad a=b=-\frac{1}{2} .
\end{array}
$$

Thus we have the following 
Theorem 1 In a generic case the normal geodesics (with the initial condition (11)) are described by the formulas (for $p_{z}>0$ ):

$$
\begin{aligned}
& x(t)=-\frac{a k^{2} \operatorname{sn}\left(\sigma_{1} b t\right) \mathrm{cn}\left(\sigma_{1} b t\right)}{\sqrt{2} \sigma_{1} b k^{\prime 2} \sqrt{1-k^{2} \mathrm{sn}^{2}\left(\sigma_{1} b t\right)}}+\frac{a \mathrm{E}\left(\operatorname{am}\left(\sigma_{1} b t\right), k\right)}{\sqrt{2} \sigma_{1} b k^{\prime 2}}+\frac{b}{\sqrt{2}} t \\
& y(t)=\frac{\mathrm{E}\left(\operatorname{am}\left(\sigma_{1} b t\right), k\right)}{\sqrt{2} \sigma_{1}}+\frac{a}{\sqrt{2}} t \\
& z(t)=\ln \operatorname{dn}\left(\sigma_{1} b t\right),
\end{aligned}
$$

where the parameters $\sigma_{1}$ and $k$ are determined by $a$ and $b\left(a b<\frac{1}{4}\right)$ via (15) and (18).

In the degenerated cases 1-4 the normal geodesics (with the initial condition (11)) are described in terms of elementary functions by the formulas (19), (21), (24), and (27).

Notice that normal geodesics in the theorem are parameterized by $a, b$. Constants $k, \sigma_{1}$ are defined by $a, b$ as explained before.

The qualitative behavior of generic normal geodesic is quite complicated. Figures 1 and 2 show parts of the geodesic spheres of radius of 0.15 and 0.25 (a scale on each figure itself; axis $z$ is exponentially scaled). A grid on the spheres corresponds to two parameters $\theta$ and $\mu$, where $\theta$ is angle of the initial vector of the geodesic with respect to the axis $x$ and $\mu$ is the initial acceleration value $x+y$ along the geodesic, i.e. $\mu$ can be interpreted as the acceleration with which the geodesic is drawn out of the starting point. In Figures $\theta$ varies from $\pi / 6$ to $5 \pi / 6$ (part of the sphere of $-\pi / 6$ to $-5 \pi / 6$ is obtained as a mirror). Parameter $\mu$ varies from -45 to 45 .

On this grid it can be seen only the qualitative behavior of a sphere with increasing radius. The drawings practically do not show those parts of spheres, which are too fast going to infinity, as well as those, which coincide to the geodesics, changing too quickly the direction. We can see that part of geodesics starting at small angle to plane $x, y$ goes to large values of coordinates $x, y$ very quickly, even for not large values of parameter $\mu$. For sufficiently large $\theta$ and mean values of $|\mu|$ geodesics deviate not too much from plane $x=y$, but if $|\mu|$ increases the deviation from this plane begins. Assuming exponential scale of axis $z$ we see that coordinate $z$ increases much more slow than $x$ and $y$. 


\section{References}

[1] Thurston, W.P. Three-dimensional manifolds, Kleinian groups and hyperbolic geometry. Bull. Amer. Math. Soc. (N.S.) 1982. V. 6, no. 3. P. 357--381.

[2] Agrachev, A., and Barilari, D.: Sub-Riemannian structures on 3D Lie groups, arXiv:1007.4970.

[3] I. Moiseev, Yu. Sachkov, Maxwell strata in sub-Riemannian problem on the group of motions of a plane, ESAIM: COCV, 16 (2010), 380-399.

[4] Yu. Sachkov, Conjugate and cut time in the sub-Riemannian problem on the group of motions of a plane, ESAIM: COCV, 16 (2010), 1018-1039.

[5] Agrachev, A.A., and Sachkov, Yu.L.: Control theory from the geometric viewpoint. Encyclopaedia of Mathematical Sciences, 87. Control Theory and Optimization, II. Springer-Verlag, Berlin, 2004.

[6] Sachkov, Yu.L.: Controllability and symmetries of invariant systems on Lie groups and homogeneous spaces. Fizmatlit, Moscow, 2007. [Russian]

[7] Boscain, U., and Rossi, F.: Invariant Carnot-Caratheodory metrics on $S^{3}, \mathrm{SO}(3), \mathrm{SL}(2)$, and lens spaces. SIAM J. Control Optim. 47 (2008), 1851-1878.

[8] Calin, O., Chang Der-Chen, and Markina, I.: SubRiemannian geometry on the sphere $\mathrm{S}^{3}$. Canad. J. Math. 61 (2009), 721-739.

[9] Gole, C., and Karidi, R.: A note on Carnot geodesics in nilpotent Lie Groups. J. Dynam. and Control Syst. 1 (1995), 535-549.

[10] Gradstein, I.S., and Ryzhik, I.M.: Tables of Integrals, Series and Products. New York: Academic, 1980.

[11] Montgomery, R.: Abnormal minimizers. SIAM J. Control Optim. 32 (1994), 1605-1620.

[12] Taimanov, I.A.: Integrable geodesic flows of non-holonomic metrics, J. Dynam. and Control Syst. 3 (1997), 129-147. 
[13] Aksenov, Ye. P, Special Functions in Celestial Mechanics. Nauka, Moscow, 1986. [Russian]

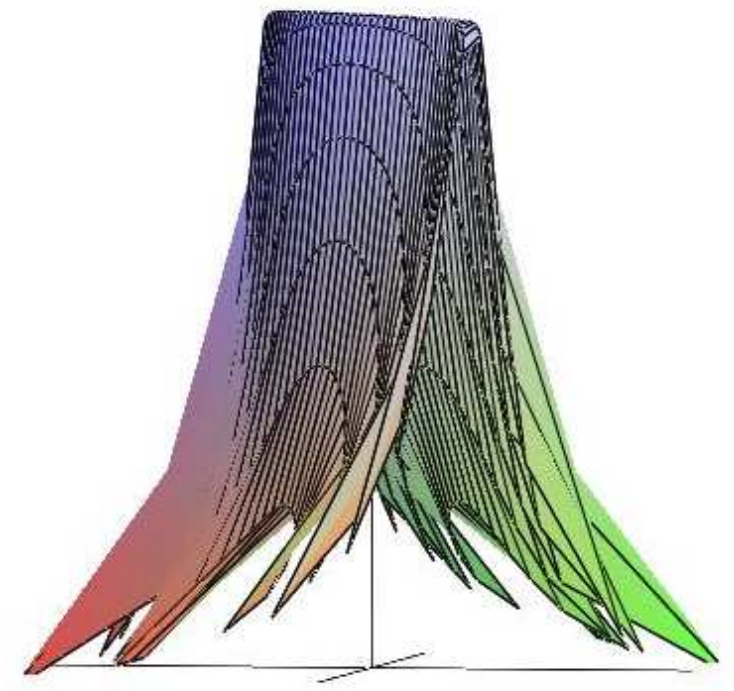

Figure 1: $r=0,15$ 


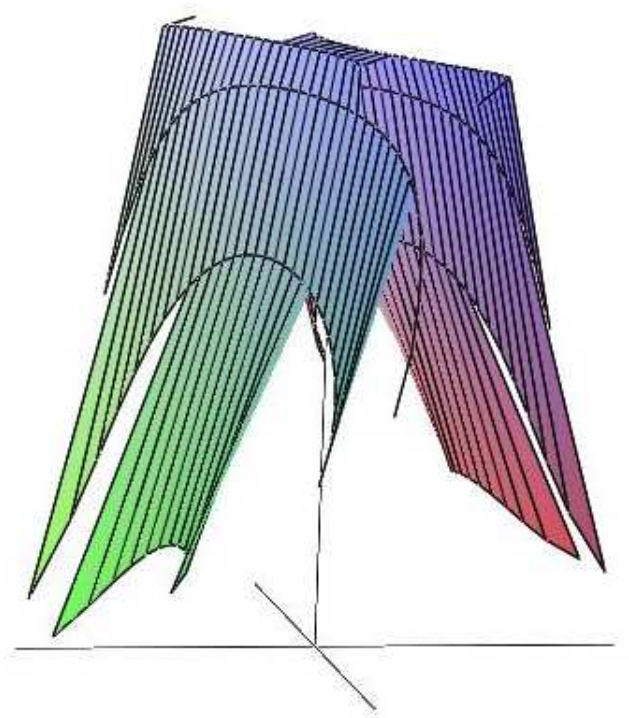

Figure 2: $\quad r=0,25$ 